\title{
Efficacy of Intrauterine Insemination in Women with Endometrioma-Associated Subfertility: Analysis Using Propensity Score Matching
}

\section{Jinlin Xie}

Northwest Women's and Children's Hospital

He Cai

Northwest Women's and Children's Hospital

Juanzi Shi

Northwest Women's and Children's Hospital

Hui Wang ( $\nabla$ wanghuiart@126.com )

Northwest Women's and Children's Hospital

\section{Research Article}

Keywords: Intrauterine insemination, endometrioma, subfertility, propensity score matching

Posted Date: October 22nd, 2021

DOI: https://doi.org/10.21203/rs.3.rs-930037/v1

License: () (1) This work is licensed under a Creative Commons Attribution 4.0 International License.

Read Full License 


\section{Abstract}

Background: Intrauterine insemination (IUI) treatment is recommended in subfertile women with AFS/ASRM stage I/II endometriosis. However, the efficacy of IUI in women with ovarian endometriomas with tubal patency is uncertain. We explored the efficacy of IUI for the treatment of endometriomaassociated subfertility.

Methods: We performed a retrospective matched cohort study using propensity matching (PSM) analysis. Subfertile couples undergoing IUI with and without ovarian stimulation between January 1,2015 , and May 30, 2020 were reviewed.

Results: After PSM, 56 women with endometrioma alone were matched to 173 patients with unexplained subfertility. The per-cycle pregnancy rate (PR) was comparable between women with endometriomaassociated subfertility ( $n=56,87$ cycles) and women with unexplained subfertility ( $n=173,280$ cycles) ( $9.2 \%$ vs. $17.9 \%$, OR $0.47 ; 95 \% \mathrm{Cl}, 0.21-1.03)$. Subgroup analyses based on IUI with or without stimulation also resulted in comparable results. A trend toward a lower cumulative pregnancy rates (CPRs) was seen in women with endometrioma $(14.3 \%, 8 / 56)$ compared with women with unexplained subfertility $(28.9 \%$, $50 / 173$ ), but the differences were not significant (OR, 0.54; $95 \% \mathrm{Cl}, 0.26-1.15)$. However, patients with endometrioma were nearly twice as likely to converse to IVF treatment compared with those without the disease (60.7\% versus $43.9 \%$; OR, $1.97 ; 95 \% \mathrm{Cl}, 1.07-3.65)$.

Conclusion: IUI may be a viable approach for subfertile women with endometrioma and no other identifiable infertility factor. More studies are needed to reassure the findings.

\section{Background}

Endometriosis is one of the most common chronic gynecologic disorders and is frequently associated with female subfertility (up to $50 \%$ subfertile women with endometriosis) [1-3]. Approximately 190 million women worldwide are currently affected by endometriosis [4] and 30-50\% of women with endometriosis are infertile [5-7]. Assisted reproductive technology (ART) frequently is used as the first-line therapy to endometriosis-associated infertility [8-10]. Guidelines of both the European Society of Human Reproduction and Embryology [9] and American Society for Reproductive Medicine [10], state that intrauterine insemination (IUI) treatment is only recommended in subfertile women with minimal-to-mild endometriosis. Werbrouck et al. reported no difference in cycle pregnancy rate between women with surgically treated minimal to mild endometriosis and women with unexplained infertility after controlled ovarian hyperstimulation and IUI program. The cumulative live-birth rate within four cycles of IUI was also comparable in women with minimal endometriosis, mild endometriosis, and unexplained infertility $(70.2 \%$, $68.2 \%, 66.5 \%$, respectively) [11].

Ovarian endometriomas are found in $17 \%-44 \%$ of women with endometriosis [12-14]. Although the exact pathophysiology of the reduced fertility is not clear, toxic content from an endometrioma may play a crucial role. Endometrioma is also usually overlap with those a more advanced stage of disease (stages III 
and IV of endometriosis according to the American Society for Reproductive Medicine (ASRM) classification).

A significant number of women with endometrioma will eventually seek ART to achieve a pregnancy, which is more often in those with reduced ovarian reserve or other identifiable infertility factor. However, for subfertile patients with presence of endometrioma alone (i.e.normal ovarian reserve and patency of fallopian tubes), is IUI treatment effective for subfertility associated with endometrioma? The optimal management often poses a clinical debate and little evidence exists to provide robust guidance to clinicians.

Considering a sequence of IUI cycles is less aggressive and less expensive than an IVF procedure, should IUI treatment be encouraged as a valuable or viable approach to achieve a natural pregnancy? The aim of the present study was to test the efficacy of IUI treatment on women with endometrioma-associated subfertility, comparing the fertility outcomes (per-cycle-pregnancy rate [PR], and cumulative pregnancy rates [CPRs] after IUI treatment in subfertile women with endometrioma and women with unexplained subfertility by using propensity matching (PSM) analysis.

\section{Methods}

\section{Study design}

We retrospectively analyzed the IUI with the husband's sperm cycles performed from January 1, 2015 to May 30, 2020. The data were extracted from the database of infertility center of Northwest Women and Children's Hospital, Xi'an, China. This study was approved by the institutional research ethics review board (2019013).

\section{Patients}

Before reproductive treatment, all subfertile couples underwent a infertility evaluation, including physical examination, transvaginal ultrasound, cycle day 2-3 serum follicle-stimulating hormone (FSH) and luteinizing hormone (LH) assays, hysterosalpingography, semen analyses and associated safety tests.

The study group consisted of all consecutive subfertile women with the presence of identified endometrioma and no other identifiable infertility factor. Inclusing criteria were failure to conceive for $\geq 12$ months, female age $\leq 40$ years, ovulation demonstrated by appropriately timed mid-luteal progesterone,bilateral tubal patency (demonstrated by hysterosalpingography or laparoscopy); normal semen variables (according to World Health Organization criteria) [15]. Patients satisfied the above criteria were included whether or not the they had prior surgical treatment for their endometrioma. The endometrioma was diagnosed either by laparoscopy or a combination of physical examination and transvaginal ultrasound.

The control group consisted of couples diagnosed with subfertility who were treated during the same period of time and who met the same inclusion criteria except with no evidence of endometrioma. Women 
were excluded from the study if they had achieved a clinical pregnancy during the previous IUI treatment. Cases from IVF with controlled ovarian stimulation conversion to IUI due to low ovarian response were also excluded.The study and control groups of patients were matched using PSM analysis.

\section{IUI Procedures and semen preparation}

IUI was performed in natural or stimulated cycles [16]. Briefly, for natural cycles, the ultrasound and serum hormone tests started on the eighth day of the cycle. When the leading follicle was $\geq 14 \mathrm{~mm}$, patients started the test for urinary LH; For stimulation cycles, ovarian stimulation was conducted by either administering letrozole or hMG (Menotropins for Injection, Livzon pharmaceutical group INC., China) or letrozole plus hMG. Ovarian response was monitored by the follicular growth and serum $\mathrm{E}_{2}$ levels starting on day 5 of stimulation, and then dose of hMG was adjusted accordingly every 1-3 days. IUI was performed 24 hours after detection of LH in the urine. If the leading follicle measured over $18 \mathrm{~mm}$ in diameter in the absence of LH in the urine, 10,000 IU of hCG were administrated and insemination was performed the next day.

Semen was prepared on the day of insemination by centrifugation on a density gradientas, as previously described [17]. All women were treated by the same two physicians (HW and JLX) with the same IUI procedure.The prepared sperm was gently inserted within $1 \mathrm{~cm}$ of the fundal extend of the uterine cavity using a soft catheter. Micronized progesterone ( $200 \mathrm{mg} /$ day) was used for 15 days after IUI.

\section{Outcome Measures}

A serum $\beta$-hCG test was performed approximately 16 days after insemination. A clinical pregnancy was diagnosed 2 weeks after a positive test by the presence of a gestational sac on ultrasound. Live birth was defined as a live-born delivery at least 24 weeks after IUI. The primary outcomes of interest were clinical pregnancy rate (PR) per cycle and cumulative pregnancy rates (CPRs) after IUI treatment. A subanalysis was performed based on IUI protocols: natural or stimulation cycle.

\section{Statistical analysis}

PSM was performed to adjust for confounding factors correlated with pregnancy outcomes. The variables in the PSM included female characteristics (i.e., age, gravidity, parity, body mass index (BMI), duration of infertility, antral follicle count (AFC), serum concentrations of FSH and LH, and male characteristics (age, semen parameters). To optimize the precision of the study, patients with endometrioma were matched to patients with unexplained infertility in a 1:3 matching ratio. The PSM allowed each endometrioma patient undergoing IUI to be matched to a unexplained infertile patient with similar characteristics.

PR-per cycle was compared between the two groups (endometrioma-associated subfertility vs. unexplained subfertility). A subanalysis was performed based on IUI protocols: natural or stimulation cycle. As one couple could have more IUI cycles we applied generalized estimating equations (GEE) that took into account this clustering. CPRs were the number of women who achieve a clinical pregnancy after one, two, or three added cycles divided by the number of women who started treatment. 
Data were expressed as mean \pm standard deviation (SD) or $n$ (\%). Descriptive data were compared by Student's T, Mann-Whitney U, Chi-squared or Fishers' exact tests when appropriate. The number of cycles since first IUI treatment were used as time parameters. The date of entry was the date of the first IUI treatment cycle. Patients were followed up to 1 year after finishing their last IUI treatment. The KaplanMeier method was used to estimate the CPRs, and comparisons were made using the log-rank test. Statistical analysis was analyzed using R (v.3.4.3; The R Foundation). $\mathrm{P}<0.05$ was considered to be significant.

Sensitivity analyses were performed on the ovarian endometrioma group versus the unexplained subfertility group with exclusion of patients without surgical diagnosis of endometrioma prior to IUI treatment.

\section{Results}

\section{Patient characteristics}

Based on inclusion criteria, 58 women with endometrioma and 880 women with unexplained infertility were available for analysis. After PSM, a total of 56 women with endometrioma were successfully matched to 173 women with unexplained infertility. No differences in age (female and male), BMI, duration of infertility, gravity and parity, AFC, basal FSH, LH levels or sperm parameters were found between the two matched groups after matching. Clinical and biological characteristics of patients before and after PSM were shown in Table 1. For women in the endometrioma group, 22 cases (39.3\%) had undergone prior surgery for endometriomas before IUI treatment and the remaining $34(60.7 \%)$ were diagnosed based on clinical and ultrasound evaluation. 
Table 1

Patient characteristics before and after propensity score matching (PSM).

\begin{tabular}{|c|c|c|c|c|c|c|}
\hline & Before PSM & & & After PSM & & \\
\hline & $\begin{array}{l}\text { Endometrioma- } \\
\text { associated } \\
\text { subfertility } \\
(\mathrm{n}=58)\end{array}$ & $\begin{array}{l}\text { Unexplained } \\
\text { subfertility } \\
(n=880)\end{array}$ & $P$ & $\begin{array}{l}\text { Endometrioma- } \\
\text { associated } \\
\text { subfertility } \\
(\mathrm{n}=56)\end{array}$ & $\begin{array}{l}\text { Unexplained } \\
\text { subfertility } \\
(n=173)\end{array}$ & $P$ \\
\hline $\begin{array}{l}\text { Maternal age } \\
\text { (years) }\end{array}$ & $30.78(4.02)$ & 30.22 (3.71) & 0.272 & 30.46 (3.72) & 30.08 (3.38) & 0.466 \\
\hline $\begin{array}{l}\text { Paternal age } \\
\text { (years) }\end{array}$ & $32.43(4.67)$ & $31.79(4.36)$ & 0.283 & $31.98(4.10)$ & $31.45(4.26)$ & 0.409 \\
\hline BMI $\left(\mathrm{kg} / \mathrm{m}^{2}\right)$ & $21.86(2.83)$ & $22.00(3.21)$ & 0.755 & $21.85(2.88)$ & $21.48(2.77)$ & 0.403 \\
\hline $\begin{array}{l}\text { Subfertility } \\
\text { time } \\
\text { (months) }\end{array}$ & $32.38(20.26)$ & $\begin{array}{l}33.12 \\
(17.40)\end{array}$ & 0.756 & $32.89(20.40)$ & $\begin{array}{l}32.46 \\
(16.01)\end{array}$ & 0.833 \\
\hline $\begin{array}{l}\text { Primary } \\
\text { infertility (\%) }\end{array}$ & $40(69.0)$ & $554(63.0)$ & 0.358 & $39(69.6)$ & $119(68.8)$ & 0.904 \\
\hline $\begin{array}{l}\text { Nulliparity } \\
(\%)\end{array}$ & 53 (91.4) & 765 (93.5) & 0.326 & $52(92.9)$ & 158 (91.3) & 0.719 \\
\hline $\mathrm{AFC}(\mathrm{n})$ & $10.00(4.78)$ & $12.75(5.58)$ & $<0.001$ & $10.21(4.72)$ & $10.87(3.79)$ & 0.293 \\
\hline $\begin{array}{l}\text { Day-3 FSH } \\
(\mathrm{mlU} / \mathrm{mL})\end{array}$ & 7.31 (1.79) & 7.04 (1.75) & 0.250 & $7.31(1.82)$ & $7.32(1.60)$ & 0.973 \\
\hline $\begin{array}{l}\text { Day-3 LH } \\
(\mathrm{mlU} / \mathrm{mL})\end{array}$ & $4.39(1.56)$ & $4.62(2.29)$ & 0.460 & $4.40(1.58)$ & $4.71(3.26)$ & 0.485 \\
\hline $\begin{array}{l}\text { Semen } \\
\text { concentration } \\
\left(10^{6} / \mathrm{mL}\right)\end{array}$ & $62.54(27.01)$ & $\begin{array}{l}62.93 \\
(27.27)\end{array}$ & 0.916 & $62.78(27.36)$ & $\begin{array}{l}62.12 \\
(26.03)\end{array}$ & 0.872 \\
\hline $\begin{array}{l}\text { Semen } \\
\text { motility (\%) }\end{array}$ & $54.26(13.77)$ & $\begin{array}{l}57.54 \\
(13.18)\end{array}$ & 0.067 & $54.44(13.93)$ & $\begin{array}{l}54.90 \\
(12.32)\end{array}$ & 0.815 \\
\hline $\begin{array}{l}\text { Normal } \\
\text { semen } \\
\text { morphology } \\
(\%)\end{array}$ & $5.64(1.66)$ & $5.48(1.70)$ & 0.493 & $5.70(1.66)$ & $5.54(1.93)$ & 0.594 \\
\hline
\end{tabular}

\section{Treatment outcomes}

The 56 women from endometrioma group underwent a total of 87 cycles of IUI (range 1-4, a mean of 1.46 attempts per patient), including 45 natural cycles and 42 cycles with stimulation. While the 173 women with unexplained infertility underwent a total of 280 cycles of IUI (range 1-5, a mean of 1.51 attempts per patient), including 152 natural cycles and 128 cycles with stimulation. 


\section{PR per-cycle}

The per-cycle clinical PR was lower in women with endometrioma ( $n=56,87$ cycles) than in the women with unexplained infertility ( $n=173,280$ cycles), though this was of borderline statistical significance (9.2\% vs. $17.9 \%$, OR $0.47 ; 95 \% \mathrm{Cl}, 0.21-1.03, \mathrm{P}=0.058)$. The subgroup analyses based on IUI with or without stimulation also resulted in comparable results (Table 2).

Table 2

Pregnancy rates in per IUI cycle treatment.

\begin{tabular}{|c|c|c|c|c|}
\hline & $\begin{array}{l}\text { Endometrioma- } \\
\text { associated } \\
\text { subfertility } \\
(n=56)\end{array}$ & $\begin{array}{l}\text { Unexplained } \\
\text { subfertility } \\
(n=173)\end{array}$ & $\begin{array}{l}\text { OR } \\
(95 \% \mathrm{Cl})\end{array}$ & $P$-value \\
\hline IUI cycles, n & 87 & 280 & & \\
\hline PR, n (\%) & $8(9.2)$ & $50(17.9)$ & $\begin{array}{l}0.47(0.21- \\
1.03)\end{array}$ & 0.058 \\
\hline \multicolumn{5}{|l|}{ Subgroups } \\
\hline Natural cycles, n & 45 & 152 & & 0.095 \\
\hline PR, n (\%) & $3(6.7)$ & $26(17.1)$ & $\begin{array}{l}0.35(0.10- \\
1.20)\end{array}$ & \\
\hline $\begin{array}{l}\text { Stimulation cycles, } \\
\text { n }\end{array}$ & 42 & 128 & & \\
\hline PR, n (\%) & $5(11.9)$ & $24(18.8)$ & $\begin{array}{l}0.59(0.21- \\
1.65)\end{array}$ & 0.310 \\
\hline \multicolumn{5}{|c|}{ IUI: intrauterine Insemination; PR: Pregnancy rate. } \\
\hline \multicolumn{5}{|c|}{$\begin{array}{l}\text { The } P \text {-value is calculated using GEE taking into account repeated measurements per individual, to } \\
\text { correct for the fact that individuals were allowed to participate more than once in this study (see } \\
\text { Statistical analysis section). }\end{array}$} \\
\hline
\end{tabular}

When compared with natural cycles, IUI with stimulation cycles seemed to result in a slightly higher PR per cycle in the group with endometriomas ( $11.9 \%$ vs. $6.7 \%, P=0.404)$, though the differences were not significant. No significant difference between the two strategies was observed in the subset of unexplained subfertility ( $18.8 \%$ vs. $17.1 \%, \mathrm{P}=0.720)$.

\section{CPRs}

In the whole population, the overall CPRs was $15.7 \%$ after one IUI cycle, $23.6 \%$ after two, $24.9 \%$ after three and $25.3 \%$ after the final attempt. The specific CPRs at the first IUI cycle in the endometrioma group compared with unexplained infertility group were, respectively, $8.7 \%$ versus $18.5 \%$; at the second cycle, $14.3 \%$ versus $32.4 \%$; For women with endometrioma-associated subfertility, all pregnancies occurred 
within the first-two cycles of IUI program. For women with unexplained infertility, three women conceived at the third attempt and one case conceived at the fifth cycle.

The CPRs in women with endometrioma-associated subfertility $(14.3 \%, 8 / 56)$ were comparable when compared with women with unexplained subfertility (28.9\%, 50/173), (OR, 0.54; 95\% Cl, 0.26-1.15, P=.108) (Fig. 1). Women with endometrioma, however, were nearly twice as likely to converse to IVF treatment compared with those without the disease (60.7\% versus $43.9 \%$, respectively; OR, $1.97 ; 95 \% \mathrm{Cl}, 1.07-3.65)$. Amongst those with endometrioma, women who had surgical removal prior to IUI had similar CPRs compared with those women with no surgical treatment ( $3 / 22$ or $13.6 \%$ versus $5 / 34$ or $14.7 \%$ ).

\section{Sensitivity analyses}

The sensitivity analyses excluding women without surgically confirmed endometrioma $(n=34)$ also resulted in comparable results. The CPRs remained slightly lower in women with cystectomy prior starting IUI treatment $(3 / 22 ; 13.6 \%)$ compared to the unexplained infertility women(50/173; $28.9 \%)$, but the difference was not significant. The number of pregnancies in each subgroup or combination, however, was small.

\section{Discussion}

\section{Main findings}

To our knowledge, this is the first report of a study focusing on the efficacy of IUI program in women with endometrioma-associated subfertility using a PSM technique. Compared with unexplained subfertile women in the matched group, the odds of per-cycle PR in subfertile women with endometriomas were 0.47 (95\% Cl, 0.21-1.03) and odds of CPRs were 0.54 (95\% Cl, 0.26-1.15). However, none of the estimations were statistically significant. We found that patients with endometriomas were nearly twice as likely to converse to IVF treatment compared with those without the disease. Subgroup analyses based on with/without prior surgery for endometrioma did not impact the outcomes. Although for women with endometrioma, stimulation cycles seemed to result in a slightly higher PR per cycle than that in natural cycles $(11.9 \%$ vs. $6.7 \%, P=0.404)$, the difference was not significant.

\section{Comparison To Other Studies}

Similar findings have been reported for moderate-to-severe endometriosis. van der Houwen et al. [18] suggested that IUI was a valuable infertility management in women with more severe endometriosis, namely moderate-to-severe endometriosis. The CPRs of $28 \%$ in patients with Stage III and Stage IV endometriosis after six subsequent IUI cycles were reported. In the current study, the CPRs in women with endometrioma were lower than that in women with unexplained infertility $(14.3 \%$ versus $28.9 \% ; P=0.108)$, but the difference was not significant. It has been noted that majority couples in the study received no 
more than 4 cycles of IUI treatment. Although it reflects daily practice, we cannot exclude the possibility that exposure to more cycles of IUI could have led to a significant difference in CPR in both groups.

The optimum cycles of IUI attempts has been a pragmatic and challenge question when counseling couples. One previous publishing, concluded that IUI for treatment of unexplained infertility should be limited to a maximum of three cycles [19]. In the current study, a significant more women with endometrioma resorted to IVF treatment compared with those without the disease. We also found that for women with endometrioma, starting from the third cycle onward, additional attempts have only rarely increased fecundability. Although these could be attributed by chance, the results also suggested that women with subfertility and endometrioma may be reassured by more active and aggressive reproductive technology. we assume this information will be helpful in the counselling process. Some selected patients may be better served by IVF procedure if they fail to conceive after two cycles of IUI. Further data on this issue are needed.

It is still debatable whether IUI with stimulation is superior to unstimulated IUI. A pragmatic randomised controlled trial failed to show any advantage of superovulation over unstimulated IUI in couples with unexplained infertility [20]. While the Cochrane view supported clomifene citrate administration and suggested it had a beneficial effect in unexplained infertility [21]. In a recent study, an increased cumulative pregnancy rate has been shown in patients receiving IUI with stimulation up to six cycles compared to three times IUI without stimulation followed by up to three times IUI with stimulation, which endorsed the cochrane view [18]. In our study, when compared to IUI without stimulation, ovarian stimulation seemed to result in a slightly higher PR per cycle in women with subfertility and endometrioma ( $11.9 \%$ vs. $6.7 \%, P=.404)$. Differences in the two protocols that are close to statistical significance, increasing the sample size of patients may reveal meaningful role of ovarian stimulation in IUI program. The rationale for ovarian stimulation in women with endometrioma has been to correct potential disorders of endocrine and ovulation, including luteinized unruptured follicle syndrome, abnormal follicular growth, and premature LH surges [22].

However, ovarian stimulation may cause some concerns among patients. Impact of ovarian stimulation on the progression of endometriosis or its recurrence was recently summarized in a systematic review [23]. According to their report, impact of ovarian stimulation on ovarian endometrioma, if present, is clinically unremarkable. In the present study with multiple simulated IUI cycles, no bleeding, infection and other related complications was reported. All these results can be used to reassure patients.

\section{Strengths And Limitations}

One of the major strengths of this study was the use of PSM analysis to achieve matched groups. Evaluating and comparing treatment strategies for subfertile women with endometrioma alone is limited by heterogeneous practices between clinicians and centers. We specifically focused on this point by matching multiple clinical covariates in patients performing IUI. Secondly, at the present study, we compared the effectiveness of IUI on women with endometrioma alone versus women with unexplained 
subfertility. The assessment was specifically confined to the impact of ovarian endometrioma alone. Definition of the "true" unexplained infertility is still controversial. Some women in the unexplained subfertility group might have undetected minimal or mild endometriosis. However, that inclusion of the control group means that our results reflect the true contributory effect of the endometrioma alone with.

This study had some limitations deserve to be underlined. The relatively small sample size of the present study, may be underpowered to detect a significant difference in reproductive outcomes. Hence the results should be interpreted with caution. Our choice of clinical protocols for the management of subfertile women with endometrioma reflects current practice in our center and the rest of the China, but the results might not be generalizable to other populations and alternative national funding strategy. The inclusion of women with endometrioma could be diagnosed by laparoscopy or imaging detection might have introduced an factor of heterogeneity. Not reliably imaging peritoneal implants of endometriosis, however, transvaginal ultrasound have shown to have good accuracy for ovarian endometioma $(95.1 \%$ $96 \%$ specificity and 93 94.7\% sensitivity) [24, 25], which is also recommended by ARSM [10]. There were few pregnancies within each subgroup (endometrioma diagnosed with/without prior surgery), sensitivity analyses indicate similar IUI treatment effects. We did not find any RCTs comparing reproductive outcomes after endometrioma cystectomy versus no treatment in women with endometrioma and addressing if IUI procedure is more successful post cystectomy compared to untreated. Future research should focus on more uniform control group and addressing the issues.

\section{Conclusion}

The choice of the best treatment for endometrioma-assocaited subfertility remains a challenge. In the current study, IUI treatment may be a viable option to achieve spontaneous pregnancy for endometrioma subfertility. These findings must be confirmed by further studies. Individualized treatment based on the patient's age, clinical condition, costs and insurances is is highlighted.

\section{Abbreviations}

AFC

Antral follicle count

ART

Assisted reproductive technology

ASRM

American Society for Reproductive Medicine

CPR

Cumulative pregnancy rates

$\mathrm{FSH}$

Follicle-stimulating hormone

GEE

Generalized estimating equations (GEE) 
IUI

Intrauterine insemination

$\mathrm{LH}$

luteinizing hormone

PSM

Propensity score matching

PR

Pregnancy rate.

\section{Declarations}

\section{Acknowledgement}

The authors thank Professor Stephan Gordts for help with linguistic modification. We are very grateful to our volunteer patients for taking part in our research. Without the efforts, this research would not have been possible.

\section{Funding}

This research received no specific grant from any funding agency in the public, commercial or not-forprofit sectors.

\section{Availability of data and material}

Data available on request corresponding author due to privacy and ethical restrictions.

\section{Author contributions}

$\mathrm{HC}$ and JZS participated on the design of the study. HW and JLX collected the data. HC conducted the statistical analyses and drafted the initial version of the manuscript. JZS provided important guidance throughout the study process and the manuscript preparation. All authors contributed in the interpretation of the data, revised the manuscript and approved the final article.

\section{Ethics approval and consent to participate}

All procedures performed in studies involving human participants were in accordance with the ethical standards of the institutional and/or national research committee and with the 1964 Helsinki declaration and its later amendments or comparable ethical standards. Informed consent was obtained from all individual participants included in the study. This study was approved by the Ethics Review Board of the Northwest Women's and Children's Hospital, Xi'an, China (2019013).

\section{Consent for publication}

Not applicable since there are no details, images, or videos relating to an individual person. 


\section{Conflict of interest}

The authors declare that there is no conflict of interest.

\section{References}

1. Eisenberg, V.H., Weil, C., Chodick, G., Shalev, V. Epidemiology of endometriosis: a large populationbased database study from a healthcare provider with 2 million members. BJOG,2018; 125: 55-62.

2. Somigliana E, Vigano P, Benaglia L, Busnelli A, Berlanda N, Vercellini P. Management of Endometriosis in the Infertile Patient. Semin Reprod Med. 2017;35(1):31-37.

3. Zondervan KT, Becker CM, Missmer SA. Endometriosis. N Engl J Med 2020;382: 1244-1256.

4. Hamdan M, Dunselman G, Li TC, Cheong Y. The impact of endometrioma on IVF/ICSI outcomes: a systematic review and meta-analysis. Hum Reprod Update. 2015 21(6):809-825.

5. Meuleman C, Vandenabeele B, Fieuws S, Spiessens C, Timmerman D, D'Hooghe T. High prevalence of endometriosis in infertile women with normal ovulation and normospermic partners. Fertil Steril 2009;92: 68-74.

6. Redwine DB. Ovarian endometriosis: a marker for more extensive pelvic and intestinal disease. Fertil Steril. 1999;72(2):310-315.

7. Missmer SA, Hankinson SE, Spiegelman D, Barbieri RL, Marshall LM,Hunter DJ. Incidence of laparoscopically confirmed endometriosis by demographic, anthropometric, and lifestyle factors. Am J Epidemiol 2004;160:784-796.

8. Kennedy S, Bergqvist A, Chapron C, D'Hooghe T, Dunselman G, Greb R et al.; ESHRE Special Interest Group for Endometriosis and Endometrium Guideline Development Group. ESHRE guideline for the diagnosis and treatment of endometriosis. Hum Reprod. 2005;20(10):2698-2704

9. Dunselman GA, Vermeulen N, Becker C, Calhaz-Jorge C, D'Hooghe T, De Bie B et al; European Society of Human Reproduction and Embryology. ESHRE guideline: management of women with endometriosis. Hum Reprod. 2014;29(3):400-412.

10. Practice Committee of the American Society for Reproductive Medicine. Endometriosis and infertility: a committee opinion. Fertil Steril. 2012;98(3):591-598.

11. Werbrouck E, Spiessens C, Meuleman C, D'Hooghe T. No difference in cycle pregnancy rate and in cumulative live-birth rate between women with surgically treated minimal to mild endometriosis and women with unexplained infertility after controlled ovarian hyperstimulation and intrauterine insemination. Fertil Steril. 2006;86(3):566-571.

12. Giudice LC, Kao LC. Endometriosis. Lancet. 2004;364(9447):1789-1799.

13. Bulun SE. Endometriosis. N Engl J Med. 2009;360(3):268-279.

14. Alborzi S, Zahiri Sorouri Z, Askari E, Poordast T, Chamanara K. The success of various endometrioma treatments in infertility: A systematic review and meta-analysis of prospective studies. Reprod Med Biol. 2019;19;18(4):312-322. 
15. World Health Organization. WHO laboratory manual for the examination and processing of human semen; Switzerland: World Health Organization; 2010. p. 1-286.

16. Mu X, Wang H, Liu PJ, Shi JZ. The interval between insemination and ovulation predicts outcome after intrauterine insemination with donor sperm (IUI-D). Int J Gynaecol Obstet. 2021 Feb 6.

17. Cai H, Gordts S, Sun J, Meng B, Shi J. Reproductive outcomes with donor sperm in couples with severe male-factor infertility after intracytoplasmic sperm injection failures. J Assist Reprod Genet. 2020;37(8):1883-1893.

18. van der Houwen LE, Schreurs AM, Schats R, Heymans MW, Lambalk CB, Hompes PG, Mijatovic V. Efficacy and safety of intrauterine insemination in patients with moderate-to-severe endometriosis. Reprod Biomed Online. 2014;28(5):590-598.

19. Aboulghar M, Mansour R, Serour G, Abdrazek A, Amin Y, Rhodes C. Controlled ovarian hyperstimulation and intrauterine insemination for treatment of unexplained infertility should be limited to a maximum of three trials. Fertil Steril. 2001;75(1):88-91.

20. Bhattacharya S, Harrild K, Mollison J, Wordsworth S, Tay C, Harrold A, McQueen D, Lyall H, Johnston L, Burrage J, Grossett S, Walton H, Lynch J, Johnstone A, Kini S, Raja A, Templeton A. Clomifene citrate or unstimulated intrauterine insemination compared with expectant management for unexplained infertility: pragmatic randomised controlled trial. BMJ. 2008,7;337:a716.

21. Hughes E, Collins J, Vanderkerckhove P. Clomiphene citrate for infertility in women. Cochrane Database Syst Rev 2000;(2):CD 000057.

22. Schenken RS, Asch RH, Williams RF, Hodgen GD. Etiology of infertility in monkeys with endometriosis: luteinized unruptured follicles, luteal phase defects, pelvic adhesions, and spontaneous abortions. Fertil Steril 1984; 41:122-130.

23. Somigliana E, Viganò P, Benaglia L, Busnelli A, Paffoni A, Vercellini P. Ovarian stimulation and endometriosis progression or recurrence: a systematic review. Reprod Biomed Online. 2019;38(2):185-194.

24. Nisenblat V, Bossuyt PMM, Farquhar C, Johnson N, Hull ML. Imaging modalities for the non-invasive diagnosis of endometriosis. Cochrane Database of Systematic Reviews 2016.

25. Goncalves MO, Siufi Neto J, Andres MP, Siufi D, de Mattos LA, Abrao MS. Systematic evaluation of endometriosis by transvaginal ultrasound can accurately replace diagnostic laparoscopy, mainly for deep and ovarian endometriosis. Hum Reprod. 2021 17;36(6):1492-1500.

\section{Figures}




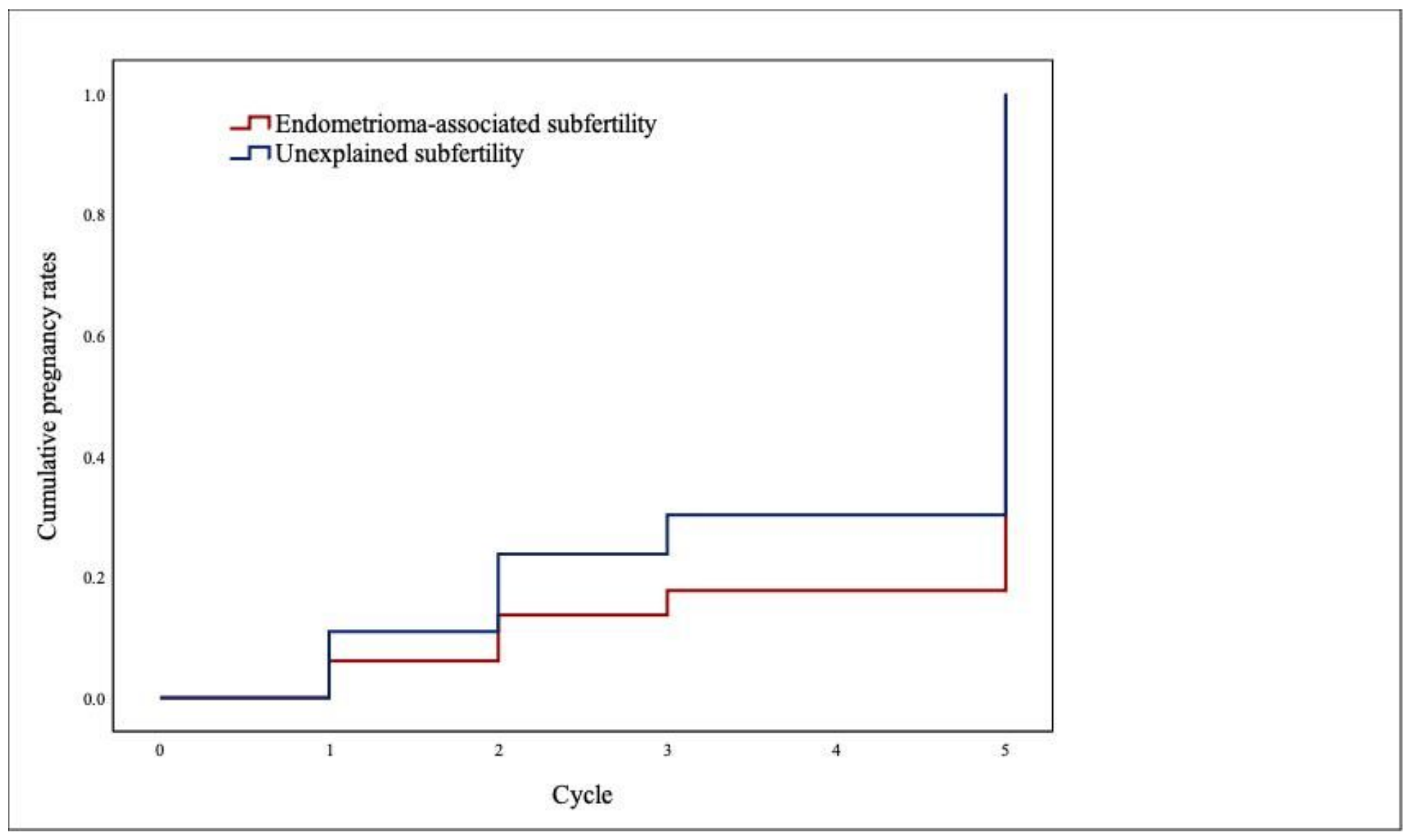

\section{Figure 1}

Kaplan-Meier curves of cumulative pregnancy rates after IUI treatment in subfertile women of the two groups $(P=.108)$. 